\title{
Empowerment, engagement e promozione della salute
}

\author{
Giuseppe Nebbioso, ${ }^{1}$ Viviana Nebbioso, ${ }^{2}$ Francesco Petrella ${ }^{3}$ \\ ${ }^{1}$ Azienda Sanitaria Locale Napoli 1 Centro; ${ }^{2}$ Università Federico II, Napoli; ${ }^{3}$ Azienda Sanitaria Locale Napoli 3 Sud, Napoli, Italia
}

I cambiamenti demografici che hanno caratterizzato l'ultimo secolo impongono un diverso approccio al concetto di promozione della salute e al rapporto medico-paziente.

In Italia tra 2008 e il 2016 il calo delle nascite è stato di circa 103.000 unità, accompagnato da un aumento dell'aspettativa di vita e da un invecchiamento della popolazione.

I cittadini con età superiore ai 65 anni sono passati dagli 11,7 milioni del 2007 ai 13,5 milioni del 2017 (22,3\% della popolazione).

L'elevato invecchiamento della popolazione è accompagnato ad un aumento di patologie croniche. Si calcola che 24 milioni di italiani (40,8 \%) sia affetto da almeno una patologia cronica, mentre il 21,5 per cento della popolazione dichiara di essere affetto da due o più patologie croniche, specie al di sopra dei 55 anni.

Tra gli ultra settantacinquenni la comorbilità si attesta al $66,6 \%$ (57,6 \% tra gli uomini e $72,9 \%$ tra le donne). ${ }^{1}$

Nel 2017 la spesa sanitaria in Italia è stata di 113.131€ milioni, dei quali il 59,4\% per cura e riabilitazione mentre il $10,4 \%$ per assistenza sanitaria a lungo termine.

La spesa sanitaria per la prevenzione delle malattie si ferma al 4,7\%.

Negli ultimi decenni ad un aumento di richiesta di sa-

Corrispondenza: Giuseppe Nebbioso, Azienda Sanitaria Locale Napoli 1 Centro, Piazza Nazionale 91, 80143 Napoli, Italia.

E-mail: giuseppe.nebbioso@gmail.com

Parole chiave: Empowerment; engagement; promozione della salute; lesioni croniche cutanee; alfabetizzazione sanitaria.

Conflitti di interesse: Gli autori dichiarano l'assenza di conflitti di interesse.

Ricevuto per la pubblicazione: 25 aprile 2020 .

Accettato per la pubblicazione: 30 novembre 2020.

This work is licensed under a Creative Commons Attribution NonCommercial 4.0 License (CC BY-NC 4.0).

${ }^{\circ}$ Copyright: the Author(s), 2021

Licensee PAGEPress, Italy

Italian Journal of Wound Care 2021; 5(1):64

doi:10.4081/ijwc.2021.64 lute si è associata una contrazione della spesa sanitaria pubblica che ha interessato tutti i paesi europei anche se in proporzioni diverse.

Nel 2017 il rapporto spesa sanitaria pubblica/PiL è stato in Italia del 6,3\% contro 9,6\% della Germania e il $9 \%$ della Francia. ${ }^{2}$

L'ultimo Def prevede, per il 2019/2020, che il rapporto tra la spesa sanitaria pubblica ed il PIL si attesti al $6,3 \%$ in contrasto con l'OMS che fissa un livello del $6,5 \%$ al di sotto del quale non si dovrebbe scendere. ${ }^{3}$

Lo squilibrio tra offerta e domanda impone una serie di riflessioni sulle possibili soluzioni di tipo socio-sanitario e politico come evidenziato nella Figura 1.

Volendo porre l'attenzione sulle tematiche sociosanitarie, le possibili vie direttici da seguire sono costituite dalla: i) Integrazione territorio-ospedale con la creazione di percorsi diagnostici-terapeutici; ii) L'impiego di nuove tecnologie come eHealth; iii) Coinvolgimento dell'ammalato e della famiglia (caregiver) nel percorso assistenziale in modo da incentivare il self care.

Queste ipotesi di lavoro possono essere sviluppate solo attraverso un adeguato finanziamento del Sistema Sanitario Nazionale (SSN) e una riflessione sulla politica sanitaria ospedalocentrica incapace di dare risposta alle cronicità.

Uno dei punti da sviluppare e incentivare è rappresentato dal coinvolgimento dell'ammalato e della famiglia (caregiver) nel percorso assistenziale in modo da incentivare il self care.

Un tale obiettivo presuppone l'empowerment del cittadino, inteso come processo attraverso cui gli individui ottengono maggiore controllo sulle decisioni e sulle azioni che riguardano la propria salute. ${ }^{4}$

In passato il cittadino/paziente aveva un ruolo passivo, di fruitore di servizi, all'interno di un sistema paternalistico della salute e non coinvolto nelle decisioni riguardanti il proprio stato di salute.

Con l'empowerment il cittadino trasforma il proprio ruolo da passivo ad attivo, partecipando al processo assistenziale di cura. Diventa capace di comprendere e partecipare al processo decisionale.

L'acquisizione dell'empowerment rende il cittadino competente engagement, più attento e sensibile alla prevenzione, più consapevole dei rischi per la salute, capace di modificare il proprio stile di vita. ${ }^{5}$ 
L'engagement nell'ambito clinico assistenziale della cronicità può essere paragonato ad un ombrello inclusivo, comprendente altri concetti quali adherence, compliance, empowerment, activation, health literacy.

L'engagement potenzia l'alleanza terapeutica tra cittadino e personale sanitario.

Il patient engagement si attiva in modo corretto ai primi segni e sintomi della malattia mettendosi in contatto tempestivamente con il sistema sanitario, contribuendo positivamente al proprio percorso di cura, gravando in maniera minore sulla spesa sanitaria. ${ }^{6}$

Uno studio su 33.000 pazienti affetti da patologia cronica ha evidenziato come un alto livello di patient engagement (impegnato e competente) permette di ridurre la spesa sanitaria fino al $21 \% .^{7}$

Uno studio, effettuato negli USA, su 2000 soggetti ospedalizzati ha evidenziato come i pazienti coinvolti nel processo di cura avessero la percezione di una qualità migliore dell'assistenza. ${ }^{8}$

Un'analisi effettuata su 3623 pazienti cronici (soggetti maggiorenni, con almeno una diagnosi di patologia cronica, residenti in Italia e fluenti nell'utilizzo della lingua italiana) ha valutato il livello di engagement mediante un questionario online auto compilato, costruito a partire da scale validate scientificamente, PHE-SCALE®.

Il 36\% degli intervistati aveva livelli di Engagement (PHE®) basso, ovvero presentava comportamenti non adeguati e consapevoli nella gestione della terapia, spesso per una mancata accettazione della malattia, evidenziando un basso livello di alfabetizzazione sanitaria. Al contrario solo il $13 \%$ presentava un pieno Engagement $(\mathrm{PHE} 囚)$ che permetteva di affrontare e gestire in maniera funzionale e attiva la propria patologia e il proprio stato di salute. ${ }^{9}$

Enpowerment e l'engagement possono essere attuati attraverso strumenti quali l'informazione sanitaria e l'educazione alla salute.

L'informazione sanitaria consiste nel fornire ai cittadini conoscenze di tipo sanitario mentre l'educazione alla salute spinge ad un uso consapevole e critico delle conoscenze sanitarie.

L'educazione alla salute è una delle attività che sono alla base della "promozione della salute" (health promotion) ed è intesa, nella Carta di Ottawa, come "il processo che consente alle persone di esercitare un maggiore controllo sulla propria salute e di migliorarla."10

La promozione della salute rappresenta un processo sociale e politico globale, che oltre ad agire sull'educazione sanitaria dell'uomo, comprende anche azioni volte a modificare i diversi determinanti della salute, quali le condizioni sociali, ambientali ed economiche, in modo da influire sulla salute non solo del singolo ma anche della collettività.

L'educazione alla salute si fonda su forme di comuni-

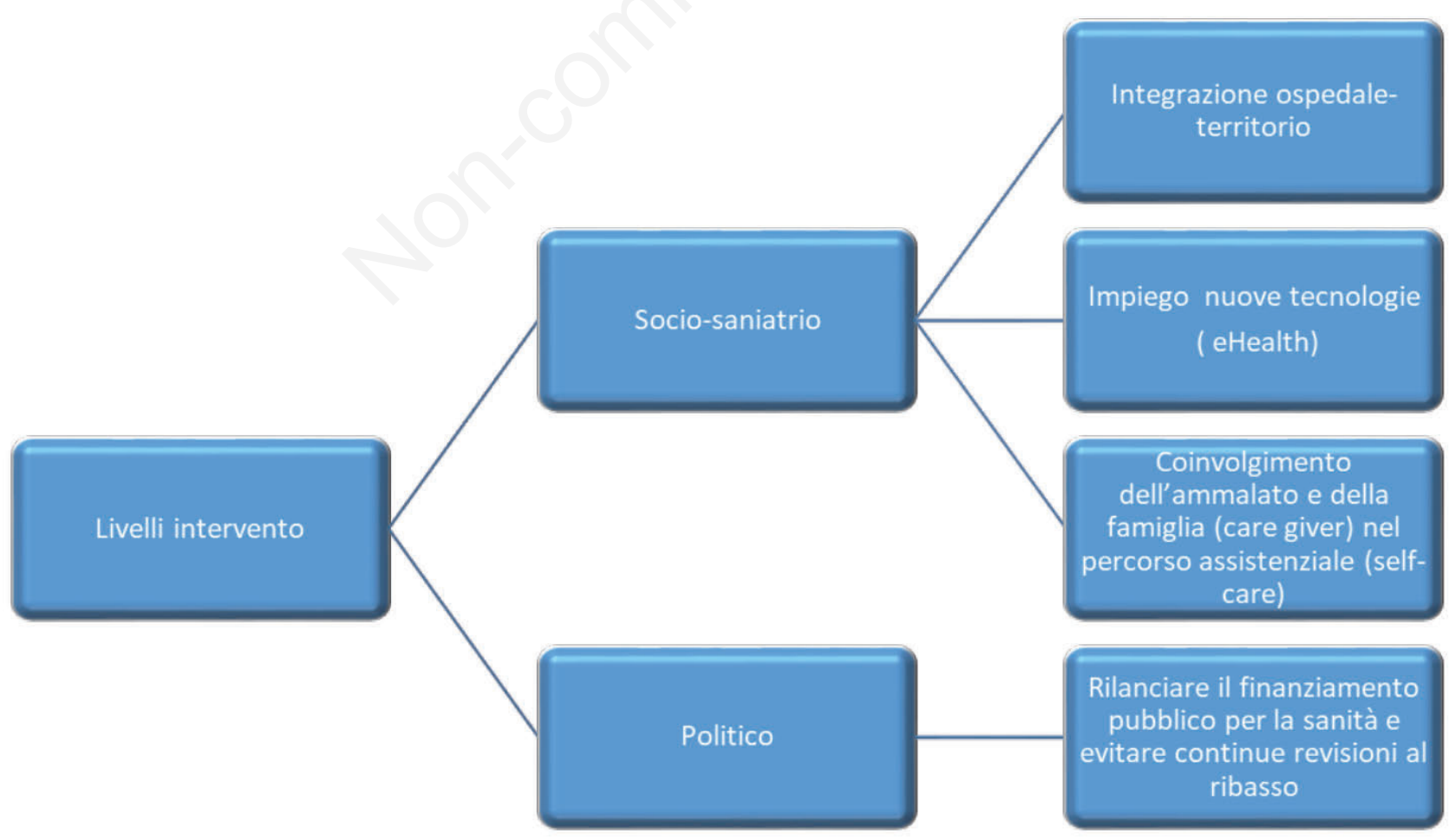

Figura 1. Livelli d'intervento in ambito socio-sanitario-politico 
cazione chiare, mirate alla tipologia del paziente/cittadino col quale ci si relaziona, al grado di cultura e alfabetizzazione sanitaria (health literacy).

Un processo così delineato si scontra con alcuni ostacoli, in particolare con la resistenza degli operatori sanitari al cambiamento del proprio modus operandi rispetto al paziente.

Infatti gli operatori devono passare da una fase prettamente prescrittiva ad una partecipativa, dal linguaggio scientifico ad uno più colloquiale.

La comunicazione è parte integrante del processo diagnostico e di cura.

Così come la corretta trasmissione dei bisogni dal paziente al medico è un requisito fondamentale per un percorso diagnostico - terapeutico ottimale, allo stesso modo una comunicazione poco efficace dal medico al paziente può condurre a livelli qualitativi inferiori di cura, complicanze, ospedalizzazione e a maggiori costi sanitari.

L'utilizzo di codici di comunicazione diversi inficia il rapporto medico-paziente, la cosiddetta alleanza terapeutica.

Per il medico e/o operatore di cura, il codice è quello della medicina basate sulle evidenze (Evidence Based Medicine, $\mathrm{EBM}$ ), per il paziente il codice è quello del vissuto e della malattia che modifica il proprio universo cognitivo, emotivo, relazionale e sociale.

La narrazione del paziente include non solo aspetti biologici ma anche psicologici, sociali, culturali, esistenziali (talvolta è fatta principalmente di questi), biografici.

La narrazione del medico, generalmente, si concentra sulle informazioni biomediche ed è fatta di concetti fisiopatologici.

Per migliorare il livello comunicativo tra paziente e medico è necessario usare un "metacodice", un sistema alla cui base vi sia la capacità di: i) riconoscere che le persone posseggono livelli di health literacy differenti; ii) usare un linguaggio semplice e comprensibile, limitando il numero dei messaggi e verificandone il livello di comprensione; iii) mettere a punto specifiche misure per accrescere il livello di health literacy predisponendo materiale informativo, brochure di educazione sanitaria.

In questo processo evolutivo in tema di comunicazione paziente -medico, l'empatia rappresenta un pilastro fondamentale, infatti, si basa sulla comprensione da parte del professionista della situazione del paziente, delle sue prospettive e dei sentimenti/emozioni che ne derivano. Il modus operandi deve essere "capire e condividere". Empatia significa dare ascolto attivo, dare spazio al paziente, dimostrare attivamente di prestargli attenzione, incoraggiandolo ad esprimersi tollerando le pause brevi o lunghe del soggetto e cercando di individuare le domande o i dubbi nascosti. ${ }^{11}$

Tempo di visita e tempo di ascolto possono influenzare in maniera positiva o negativa l'alleanza terapeutica tra medico e paziente.

Il tempo di visita al paziente è differente nelle diverse nazioni europee in Italia è di circa 16 minuti contro i 17 della Svizzera e i 5 dell'Austria (Figura 2). ${ }^{12}$

Il paziente lamenta, spesso, la "poca sensibilità" all'ascolto e la scarsa empatia che i medici hanno nei loro

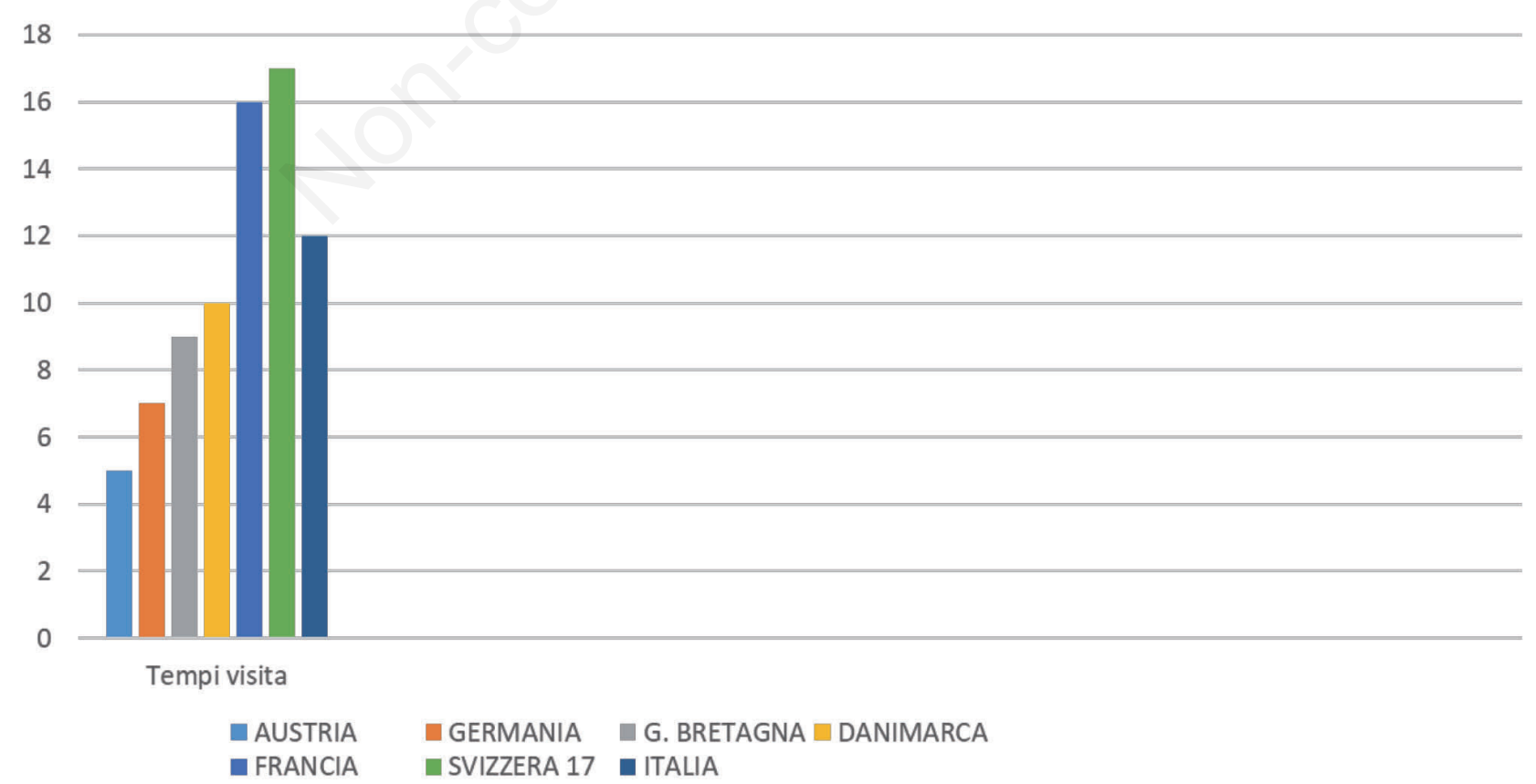

Figura 2. Tempi di visita in alcuni paesi europei. 
confronti così come la tendenza ad essere interrotti dopo pochi secondi dall'inizio del loro racconto con successiva difficoltà a riprendere il filo del discorso. ${ }^{13}$

Nel settore del wound care il processo di empowerment e engagement assume un'importanza fondamentale in quanto i pazienti affetti da Lesioni Croniche Cutanee (LCC) non hanno alcuna informazione e formazione sulla loro patologia.

Nel Quality of Life Study in Wound Care, studio condotto su 450 pazienti con LCC, si denotava come il 74\% degli intervistati avesse un basso grado di scolarizzazione ed era rappresentato soprattutto da anziani in condizioni economiche insoddisfacenti. ${ }^{14}$

Un ruolo non trascurabile hanno le associazioni dei pazienti che rappresentano il trait d'union ufficiale tra la voce dei pazienti e il mondo delle istituzioni. In particolare il paziente con la propria esperienza di malattia, le conoscenze acquisite e le difficoltà superate farà da guida agli altri pazienti affetti da specifica condizione patologica

Nell'ottica di informazione e formazione del paziente e dei suoi familiari, seguendo le indicazioni del "Chronic Care Model" nell'ambito del progetto "PAUC di alfabetizzazione sanitaria nel wound care" condotto dall'Associazione Italiana Ulcere Cutanee (AIUC) e dalla SIMITU (Associazione per i diritti dei pazienti affetti da lesioni croniche cutanee) è stato avviato un percorso di formazione dei caregiver rivolto ai familiari di pazienti affetti da LCC, volendo dimostrare come un tale percorso possa ridurre gli accessi sanitari e migliorare la qualità di vita del paziente.

Le line di indirizzo sono state due, una più generica rivolta a tutti i pazienti e ai familiari di questi ultimi basata sulla distribuzione di opuscoli informativi e sulla possibilità di collegarsi ai siti delle associazioni in questione dove è possibile avere informazioni sulla patologia, sulla prevenzione e sui centri di cura di riferimento.

Una seconda linea rivolta ai familiari, ai caregiver, di pazienti affetti da ulcere venose nei quali dopo aver valutato il grado di alfabetizzazione di base, attraverso un colloquio con gli operatori, con un linguaggio semplice e comprensibile, è stata spiegata la tipologia di lesione che dovevano affrontare, la causa e, dopo training formativo, come medicare e bendare il paziente.

$\mathrm{Al}$ primo accesso post formativo è stata valutata la capacità di medicazione e bendaggio valutando la comparsa di segni clinici quali edema dell'arto, strie cutanee e sintomi quali dolore e/o bruciore.

1 percorso formativo basato sul PAUC ha dato risultati positivi facendo sì che i pazienti protessero essere medicati, presso il nostro centro, una volta a settimana effettuando l'altra medicazione a domicilio. Nessun paziente lamentava dolore e/o bruciore in seguito alla medicazione e al bendaggio. Da segnalare che in seguito alle prime medicazioni sulle gambe di alcuni pazienti erano presenti delle strie cutanee dovute alla maggiore trazione data alla benda in al- cuni punti, casi risolti apponendo al di sotto del bendaggio del cotone di Germania. ${ }^{15}$

Appare evidente come, anche nel wound care, il miglioramento del rapporto medico-paziente passi attraverso nuove forme di ascolto e comunicazione e attraverso nuove prerogative quali: i) Competenza a trattare risolvere nel modo migliore determinate problematiche sanitarie; ii) $\mathrm{Ca}$ pacità di adattamento a relazionarsi alla tipologia di paziente e al suo vissuto; iii) Capacità di aggiornamento, mediante acquisizione di nuove conoscenze atte a migliorare le proprie performance; iv) Auto riflessione critica, come processo strutturato che facilita il riflettere su sé stessi e sul proprio operato.

Compito delle società scientifiche dovrà essere quello di contribuire allo sviluppo di tale processo, attraverso interventi formativi e motivazionali da concordare e sviluppare con il sostegno di altri settori della società, in particolare modo quello politico e sociale.

\section{BIBLIOGRAFIA}

1. Sistema statistico nazionale, Istituto nazionale di statistica, annuario 2019.

2. Eurostat - Statistiche europee 2019.

3. Rapporto sanità OCSE 2019.

4. Rappaport J. Terms of empowerment/exemplars of prevention: Toward a theory for community psychology. Am J Comm Psycol 1987;15:121-48.

5. Wallerstein $\mathrm{N}$. What is the evidence on the effectiveness of empowerment to improve health? Copenhagen, Denmark: World Health Organization (WHO), Regional Office for Europe, Health Evidence Network; 2006.

6. Kripalani S, Weiss BD. Teaching about health literacy and clear communication. J Gen Intern Med 2006;21:888-90.

7. Fisher ES, Shortell SM, Savitz LA. Implementation Science A Potential Catalyst for Delivery System Reform. JAMA 2016;315:339-40.

8. Weingart SN, Zhu J, Chiappetta L, et al. Hospitalized patients' participation and its impact on quality of care and patient safety. Int J Qual Health Care 2011;23:269-77.

9. Report engagement monitor 2019. Consumer and Health Engagement Research Center. Università Cattolica del Sacro Cuore. Novembre 2019.

10. Ottawa Charter for Health Promotion. Geneva, Svizzera: WHO; 1986.

11. Aragona M, Puzella A. How empathy for pain changes in the neurosciences: reciprocal influences between conceptual and experimental issues. A critical review of functional neuroimaging studies. Giorn Ital Psicopat 2010;16:309-20.

12. Irvin G, Neves AL, Dambha-Miller H et al. International variations in primary care physician consultation time: a systematic review of 67 countries. BMJ 2017;7:e017902.

13. Marvel MK. Soliciting the patient's agenda: have we improved? JAMA 1999;281:283-7.

14. Nebbioso G, Albanese C, Bonat F, et al. Qualità della vita nello studio Wound Care. Rapporto conclusivo. Ital J Wound Care 2017;1:10-3.

15. Nebbioso G, et al. Alfabetizzazione sanitaria e wound care. Atti XV Congresso Nazionale AIUC. Napoli 2019. 